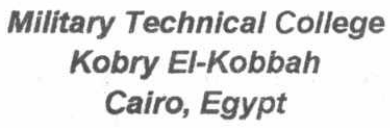

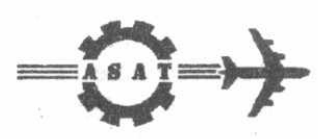

$10^{\text {th }}$ International Conference On Aerospace Sciences\& Aviation Technology

\title{
SUPPRESSION OF COHERENT INTEREFERENCES USING UNIFORM LINEAR ANTENNA ARRAY
}

\author{
Assist. Prof. K. A. Elbarbary \& Assist. Prof. E. E. Azzouz \\ Egyptian Armed Forces
}

\begin{abstract}
This paper is concerned with introducing a system for reducing the effect of coherent interference sources. This system consists of two units connected with an adaptive antenna array. The first is concerned with the direction of arrival (DOA) estimation of incident signals. The second is used for generating adaptive nulls in the direction of undesired interference. Modified Multiple Signal Classification (Modified MUSIC) algorithm is used for estimating the DOA. Also, an adaptive method for generating nulls in the direction of undesired interferences is introduced and it is denoted by Complementary Transformed Minimum Variance (CTMV) beam former. Computer simulations indicate that the proposed system is robust in presence of coherent and noncoherent interferences.
\end{abstract}

Key Words: Adaptive Array Processing, Direction of Arrival Estimation, Interference Cancellation 


\section{1- Introduction}

Conventional signal detection systems are subjected to the requirement of high signal to noise ratio (SNR) because of the presence of undesired noise in the signal environment. This noise may enter the system either through the sidelobes or the mainlobe of the antenna beam pattern. Signal reception using an array of sensor elements introduces an attractive solution to severe problems of signal detection and estimation because an array offers a means of overcoming the directivity and beam width limitations of a single sensor element. Generally, array processing is used to achieve some requirements such as: enhancing the reception performance by simultaneously suppressing any undesirable interference and preserving the desired signal, determining the number of sources and their directions, fine and high resolution in angles estimation, and tracking several sources simultaneously. Interference suppression is obtained by appropriate null steering in the beam pattern to reduce side-lobe levels in the directions of interference sources, while the mainlobe level is maintained in the direction of the desired signal. An adaptive array system relies heavily on spatial characteristics of the received signal to improve the interference suppression capability of the receiving system.

However, most of the interference suppression beam formers assume that the interference source is uncorrelated with the desired signal. This is not the situation in some cases such as repeater jamming signals, which are strongly coherent with the desired signal. In such situations of coherent interference signals, the conventional beam former fails to estimate the direction of arrival of either the desired signal or the interference one.

In this paper, we introduce a combination of a modified version of the MUSIC algorithm and an adaptive beam former based on the complementary transformed minimum variance (CTMV) algorithm. The modified MUSIC algorithm is used for estimating the DOA of the desired signal in the presence of coherent interference. The adaptive beam former is used for null steering in the direction of the undesired interference. The proposed system shows robust performance in the presence of both coherent and uncorrelated interference signals.

The paper is organized as follows: In the following section, the proposed system for interference nulling is presented. In the proposed system, a modified version of the MUSIC algorithm is introduced for direction of arrival estimation, and the CTMV is presented for generating nulls in the directions of undesired interferences. Computer simulations and performance evaluations for the proposed system in different cases are presented in section 3. Finally, the paper is concluded in section 4 .

\section{2- Proposed System For Coherent Interference Cancellation}

The proposed system for interference cancellation is shown in Fig. 1. It consists of an array antenna connected to two subsystems; the first is concerned with the estimation of the angles of arrival for the impinging signals, and the second steers nulls in the directions of the undesired interference signals. It is worth noting that the DOA of the desired signal is assumed to be a prior known. In Fig. 1, the input signal enters the DOA estimation subsystem, where the covariance matrix is estimated and given to the modified MUSIC algorithm. The spatial spectrum is evaluated and is fed to the peak detector unit, which extracts the peaks of the spatial spectrum then send their angle values to the nulling subsystem that can successfully steer nulls in the directions of the interference. The modified MUSIC algorithm is used for DOA estimation due to its capability to estimate both uncorrelated and coherent signals. This capability is due to the fact that the modified 
MUSIC algorithm consists of spatial smoothing and traditional MUSIC algorithm. The CTMV method is presented to deal with coherent interference. The nulling subsystem tries to minimize the output power of the system until it reaches a certain threshold at which the null will be at the direction of the undesired interference.

\section{1- Modified MUSIC Algorithm for DOA Estimation}

There are three approaches for the DOA estimation of a signal [1]. The first approach is the conventional approach, the second is the subspace approach, and the third is the parametric approach. The conventional approach depends on maximization of output power of the array system. The subspace approach exploits the eigen-decomposition of the array covariance matrix [2]. The parametric approach is concerned with the maximization of a criterion with respect to a certain parameter. In each approach, several methods are applicable. In this paper, we choose the subspace approach for DOA estimation and especially the modified MUSIC algorithm [3]. The subspace approach won popularity due to their high-resolution properties and the capability to resolve closely spaced sources. Suppose that there is a planar wavefront impinging a uniform linear array (ULA) antenna of $M$ elements with equal spacing, $\Delta=\lambda / 2$, and $\lambda$ is the wavelength. Let $\phi$ be the direction of arrival of the planar wavefront measured with respect to the bore line of the elements. If the first element is taken as a reference point, the phase difference between the mth element and the first one is given by

$$
\theta_{m}=\frac{2 \pi}{\lambda} \Delta m \sin (\phi) \quad, \mathrm{m}=0,1,2, \ldots, \mathrm{M}-1
$$

So if the complex envelope of the narrow band signal impinging to the first element is $\mathbf{s}_{0}$ (t), the signal at the $\mathrm{m}^{\text {th }}$ element will be

$$
\mathrm{s}_{\mathrm{m}}(\mathrm{t})=\mathrm{s}_{0}(\mathrm{t}) \mathrm{e}^{\mathrm{j} \theta_{\mathrm{m}}}
$$

So we can denote the signal at the array elements as

$$
\mathrm{x}(\mathrm{t})=\mathrm{a}(\phi) \mathrm{s}_{\mathrm{o}}(\mathrm{t})
$$

Where $\mathrm{a}(\phi)$ is the steering vector and it is given by

$$
\mathrm{a}(\phi)=\left[\begin{array}{lllll}
1 & \mathrm{e}^{\mathrm{j} \theta_{1}} & \mathrm{e}^{\mathrm{j} \theta_{2}} & \cdots & \mathrm{e}^{\mathrm{j} \theta_{M-1}}
\end{array}\right]^{\mathrm{T}}
$$

Also, assuming that there are $\mathbf{D}$ signals incident on the array, the received signal at the input of the antenna array can be expressed as a linear combination of the $\mathbf{D}$ incident waveforms and noise; i.e.

$$
X(t)=\sum_{d=0}^{D-1} a\left(\phi_{d}\right) s_{d}(t)+n(t)
$$

In a matrix form, $\mathbf{X}(\mathbf{t})$ can be expressed by

where

$$
X(t)=A(\phi) S(t)+n(t)
$$

and

$$
\mathrm{A}(\phi)=\left[\mathrm{a}\left(\phi_{0}\right) \mathrm{a}\left(\phi_{1}\right) \mathrm{a}\left(\phi_{2}\right) \ldots \mathrm{a}\left(\phi_{\mathrm{D}-1}\right)\right]
$$

$$
S(t)=\left[S_{0}(t) S_{1}(t) S_{2}(t) \ldots . S_{D-1}(t)\right]^{T}
$$


From equation (6) it can be noticed that the received signal vector $\mathbf{X}(\mathbf{t})$ is a linear combination of $\mathrm{A}(\phi)$ and $\mathrm{S}(\mathrm{t})$. Thus the input covariance matrix $\mathbf{R}_{\mathbf{x}}$ can be expressed as

$$
R_{X}=E\left[X(t) X^{H}(t)\right]=A(\phi) E\left[S(t) S^{H}(t)\right] A^{H}(\phi)+E\left[n(t) n^{H}(t)\right]
$$

writing the previous equation in quadratic form

$$
\mathrm{R}_{\mathrm{x}}=\mathrm{A}(\phi) \mathrm{R}_{\mathrm{s}} \mathrm{A}^{\mathrm{H}}(\phi)+\sigma_{\mathrm{n}}{ }^{2} \mathrm{I}
$$

Where $\mathbf{R}_{\mathbf{s}}$ is the covariance matrix of the source signals.

Let $\mathbf{D}_{1}$ denotes the rank of the matrix $\mathbf{R}_{\mathbf{s}}$. If some sources of the signals are coherent, then $\mathbf{D}_{1}<\mathbf{D}$, but if all sources are uncorrelated then $\mathbf{D}_{1}=\mathbf{D}$. The eigen-decomposition of $\mathbf{R}_{\mathbf{x}}$ is given by [2]

$$
\mathrm{R}_{\mathrm{x}}=\sum_{\mathrm{m}=1}^{\mathrm{M}} \lambda_{\mathrm{m}} \mathrm{e}_{\mathrm{m}} \mathrm{e}_{\mathrm{m}}^{\mathrm{H}}
$$

Where $\lambda_{\mathrm{m}}$ are the eigen values of $\mathbf{R}_{\mathbf{x}}$ ordered as $\lambda_{0}>\lambda_{2}>\ldots . .>\lambda_{\mathrm{M}-1}$, and $\mathbf{e}_{\mathrm{m}}$ are the corresponding eigen vectors.

We know that the eigen values of $\mathbf{R}_{\mathbf{x}}, \lambda_{0}, \lambda_{2}, \ldots \ldots \ldots \lambda_{\mathrm{M}-1}$, are satisfying

$$
\begin{gathered}
\left|\mathrm{R}_{\mathrm{x}}-\lambda_{\mathrm{m}} \mathrm{I}\right|=0 \\
\left|\mathrm{~A}(\phi) \mathrm{R}_{\mathrm{s}} \mathrm{A}^{\mathrm{H}}(\phi)+\sigma_{\mathrm{n}}{ }^{2} \mathrm{I}-\lambda_{\mathrm{m}} \mathrm{I}\right|=\left|\mathrm{A}(\phi) \mathrm{R}_{\mathrm{s}} \mathrm{A}^{\mathrm{H}}(\phi)-\left(\lambda_{\mathrm{m}}-\sigma_{\mathrm{n}}{ }^{2}\right) \mathrm{I}\right|=0
\end{gathered}
$$

Which means that the matrix $\left[\mathbf{A}(\phi) \mathbf{R}_{\mathbf{s}} \mathbf{A}^{\mathrm{H}}(\phi)\right]$ has the eigen values, $\mathbf{v}_{\mathbf{m}}$, given by

$$
\mathrm{v}_{\mathrm{m}}=\lambda_{\mathrm{m}}-\sigma_{\mathrm{n}}^{2}
$$

Since $\mathrm{A}(\phi)$ is composed of steering vectors which are linearly independent, it has full column rank. Thus the matrix $\left[\mathbf{A}(\phi) \mathbf{R}_{\mathbf{s}} \mathbf{A}^{\mathbf{H}}(\phi)\right]$ has $\mathbf{M} \times \mathbf{M}$ dimensions, and it has rank $\leq \mathbf{D}$, which means that there are at least $\mathbf{M}-\mathbf{D}$ of the eigen values of $\left[\mathbf{A}(\phi) \mathbf{R}_{\mathbf{s}} \mathbf{A}^{\mathbf{H}}(\phi)\right]$ equal to zeros [2]. Thus, from (12) we get $\mathbf{K}=\mathbf{M}-\mathbf{D}$ of eigen values of $\mathbf{R}_{\mathbf{x}}$ are equal to the noise variance $\sigma_{\mathrm{n}}^{2}$

$$
\lambda_{\mathrm{D}}, \ldots \lambda_{\mathrm{M}-1}=\sigma_{\mathrm{n}}^{2}
$$

So computing the multiplicity $(\mathbf{K})$ of the smallest eigen value, one can estimate the number of signals $\mathbf{D}=\mathbf{M}-\mathbf{K}$. For eigen vectors associated with the $\mathbf{M}-\mathbf{D}$ smallest eigen values, we have

$$
\left(\mathrm{R}_{\mathrm{x}}-\lambda_{\mathrm{m}} \mathrm{I}\right) \mathrm{e}_{\mathrm{m}}=\mathrm{A}(\phi) \mathrm{R}_{\mathrm{s}} \mathrm{A}^{\mathrm{H}}(\phi) \mathrm{e}_{\mathrm{m}}+\sigma_{\mathrm{n}}^{2} \mathrm{I}-\sigma_{\mathrm{n}}^{2} \mathrm{I}=0
$$

Thus,

$$
\mathrm{A}(\phi) \mathrm{R}_{\mathrm{s}} \mathrm{A}^{\mathrm{H}}(\phi) \mathrm{e}_{\mathrm{m}}=0
$$

Since $A$ is full rank and $R_{s}$ is nonsingular, this implies

$$
\mathrm{A}^{\mathrm{H}}(\phi) \mathrm{e}_{\mathrm{m}}=0
$$

This relation means that the eigen vectors associated with $\mathbf{M}-\mathbf{D}$ smallest eigen values are orthogonal to the D steering vectors for $\mathrm{A}(\phi)$.

So we can estimate the steering vectors $\mathrm{A}(\phi)$ associated to the received signals by finding the vectors which are most nearly orthogonal to the eigen vectors associated to the eigen values of $R_{\mathbf{x}}$ that are approximately equal to $\sigma_{n}{ }^{2}$. From the orthogonality property of the 
steering vectors corresponding to the signal components and the noise subspace vectors we can write that

$$
\mathrm{a}^{\mathrm{H}}(\phi) \mathrm{E}_{\mathrm{n}} \mathrm{E}_{\mathrm{n}}^{\mathrm{H}} \mathrm{a}(\phi)=0
$$

Where $\mathbf{E}_{\mathbf{n}}$ is the noise subspace spanned by the eigen vectors, $\mathbf{e}_{\mathrm{m}}$, which satisfies Eqn. (16). Then the DOAs of the multiple incident signals can be estimated by locating the peaks of the MUSIC spectrum that is given by [3]

$$
\mathrm{f}_{\text {music }}(\phi)=\frac{\mathrm{a}^{\mathrm{H}}(\phi) \mathrm{a}(\phi)}{\mathrm{a}^{\mathrm{H}}(\phi) \mathrm{E}_{\mathrm{n}} \mathrm{E}_{\mathrm{n}}^{\mathrm{H}} \mathrm{a}(\phi)}
$$

However, in MUSIC algorithm we supposed that there were D signals impinging $\mathbf{M}$ element array antenna and we found that this method failed in the case of coherent signals. Assume that there are $\mathbf{D}$ signals at the input of an array antenna of $\mathbf{M}$ elements. The first two signals $\mathbf{s}_{0}(\mathbf{t})$ and $\mathbf{s}_{1}(\mathbf{t})$ are supposed to be coherent and the other D-2 signals are uncorrelated. In this case, the signal vector at the array elements is given by

$$
\mathrm{X}(\mathrm{t})=\mathrm{A}(\phi) \mathrm{S}(\mathrm{t})+\mathrm{n}(\mathrm{t})
$$

Because of the coherency between $s_{0}(t)$ and $s_{1}(t)$ the source signal vector will be given by

$$
\mathrm{S}^{\mathrm{T}}(\mathrm{t})=\left[(1+\alpha) \mathrm{s}_{0}(\mathrm{t}) \mathrm{s}_{2}(\mathrm{t}) \cdots \mathrm{s}_{\mathrm{D}-1}(\mathrm{t})\right]
$$

Thus, the steering vectors of the array will be given by

$$
\mathrm{A}(\phi)=\left[\begin{array}{ll}
\mathrm{a}\left(\phi_{0}\right)+\alpha \mathrm{a}\left(\phi_{1}\right) & \mathrm{a}\left(\phi_{2}\right) \cdots \mathrm{a}\left(\phi_{\mathrm{D}-1}\right)
\end{array}\right]
$$

The input covariance matrix $R_{x}$ is

$$
\mathrm{R}_{\mathrm{x}}=\mathrm{A}(\phi) \mathrm{R}_{\mathrm{s}} \mathrm{A}^{\mathrm{H}}(\phi)+\sigma^{2} \mathrm{I}
$$

From equations (20) and (21) it is noticed that the antenna array sees the two coherent signals $\mathbf{s}_{\mathbf{0}}(\mathbf{t})$ and $\mathbf{s}_{\mathbf{1}}(\mathbf{t})$ as one signal coming from the direction $\left[\mathrm{a}\left(\phi_{0}\right)+\alpha \mathrm{a}\left(\phi_{1}\right)\right]$. So the source covariance matrix $\mathbf{R}_{\mathbf{s}}$ becomes a $((D-1) \times(D-1))$ matrix. Thus, $\mathrm{A}(\phi) \mathrm{R}_{\mathrm{s}} \mathrm{A}^{\mathrm{H}}(\phi)$ is a $(\mathrm{M} \times \mathrm{M})$ matrix with rank $\mathrm{D}_{\mathbf{1}}=\mathbf{D}-\mathbf{1}$. Also $\mathrm{R}_{\mathrm{x}}$ is a $(\mathrm{M} \times \mathrm{M})$ matrix with the same rank. So there are $\left(M-D_{1}\right)$ of the eigen values of $\mathrm{R}_{\mathrm{x}}$ has the value $\sigma_{n}{ }^{2}$, and their corresponding (M-D $)$ eigen vectors are orthogonal to the columns of $A(\phi)$. These eigen vectors span the noise subspace $\mathbf{E}_{\mathbf{n}}$. Remembering that the first column of $\mathrm{A}(\phi)$ does not denote a real direction. Thus the noise subspace $\mathbf{E}_{\mathbf{n}}$ is orthogonal to

$\phi_{0}+\alpha \phi_{1}$ instead of $\phi_{0}$ and $\phi_{1}$. So the MUSIC spectrum has peaks at DOAs of just the uncorrelated signals. To avoid the cancellation in the directions of both the desired signal and the interference, the nominator of the spectrum of the modified MUSIC is replaced by unity. Thus, the spectrum of the modified MUSIC is given by [3]:

$$
\mathrm{f}_{\text {music }}(\phi)=\frac{1}{\mathrm{a}^{\mathrm{H}}(\phi) \mathrm{E}_{\mathrm{n}} \mathrm{E}_{\mathrm{n}}^{\mathrm{H}} \mathrm{a}(\phi)}=\frac{1}{\mathrm{a}^{\mathrm{H}}(\phi) \mathrm{Ua}(\phi)}
$$

\section{2-Complementary Transformed Minimum Variance (CTMV) Algorithm For Interference Cancellation \\ Conventional adaptive beam formers break down in case of coherent interference as a result of desired signal cancellation. So, it is necessary to decouple the desired signal from}


other coherent interferences in order to avoid mutual cancellation. To achieve this, the CTMV adaptive array processor performs a transformation, $\mathrm{T}$, to remove the desired signal and retain the coherent interference using DOAs estimates. This transformation is constructed to minimize the difference between the original and transformed data.

The scenario considered herein involves a single desired source, $\mathbf{J}$ coherent interferers, and $\mathbf{K}$ uncorrelated interferers, all assumed to be narrow band with the same center frequency. These sources are in the far field of an array of $\mathbf{M}$ elements characterized by a known steering vector structure. Thus, the input signal can be expressed by

$$
\mathrm{x}=\mathrm{s}_{0}+\sum_{\mathrm{i}=1}^{\mathrm{J}} \mathrm{s}_{\mathrm{i}} \mathrm{a}\left(\phi_{\mathrm{i}}\right)+\sum_{\mathrm{i}=\mathrm{J}+1}^{\mathrm{J}+\mathrm{K}} \mathrm{s}_{\mathrm{i}} \mathrm{a}\left(\phi_{\mathrm{i}}\right)+\mathrm{n}
$$

Generally, the CTMV beam former algorithm computes the transformation, T, which is obtained as the solution of the following optimization problem

$$
\begin{aligned}
& \min _{T} \operatorname{tr}\left\{(T-I) R_{x}(T-I)^{H}\right\} \\
& \text { subject to: } T \hat{A}_{c}=\hat{B}_{c}
\end{aligned}
$$

Where

$$
\hat{A}_{\mathrm{C}}=\left[\mathrm{a}\left(\hat{\phi}_{0}\right), \mathrm{a}\left(\hat{\phi}_{1}\right), \cdots, \mathrm{a}\left(\hat{\phi}_{\mathrm{J}}\right)\right]
$$

and

$$
\hat{\mathrm{B}}_{\mathrm{C}}=\left[0_{\mathrm{M}}, \mathrm{a}\left(\hat{\phi}_{1}\right), \cdots, \mathrm{a}\left(\hat{\phi}_{\mathrm{J}}\right)\right]
$$

So, $\mathrm{T}$ is given by [4]

$$
T=I-\left(\hat{A}_{C}-\hat{B}_{C}\right)\left(\hat{A}_{C} \bar{R}_{x}^{-1} \hat{A}_{C}\right)^{-1} \hat{A}_{C}^{H} \bar{R}_{x}^{-1}
$$

The modified covariance matrix, $\stackrel{\mathrm{R}}{\mathrm{R}}_{\mathrm{x}}$, is defined as follows:

$$
\stackrel{\tilde{R}}{x}_{x}=\mathrm{TR}_{\mathrm{x}} \mathrm{T}^{\mathrm{H}}-\sigma_{\mathrm{n}}^{2} \mathrm{TT}^{\mathrm{H}}+\sigma_{\mathrm{n}}^{2} \mathrm{I}
$$

The optimum weight vector $\mathrm{W}$ which remove both coherent and non coherent interference is given by:

$$
\mathrm{W}=\frac{1}{\mathrm{a}^{\mathrm{H}}\left(\hat{\phi}_{0}\right) \tilde{\mathrm{R}}_{\mathrm{x}}^{-1^{\mathrm{H}}} \mathrm{a}\left(\hat{\phi}_{0}\right)} \tilde{\mathrm{R}}_{\mathrm{x}}^{-1} \mathrm{a}\left(\hat{\phi}_{0}\right)
$$

\section{3- Computer Simulation and Performance Evaluations}

Computer simulations have been carried out to evaluate the performance of the proposed system. It is assumed that, the number of snapshots, $\mathrm{N}$, is 128 samples from the combined 
signals. Also, the combined signal is composed uf three sources (desired signal, a coherent interference and a non coherent interference) with equal powers of $10 \mathrm{~dB}$ relative to back ground noise, and arriving angles $\left[0^{\circ},-25^{\circ}, 35^{\circ}\right]$ respectively to the input of an 11 -element uniform linear array. The spatial spectrum using the traditional MUSIC is shown in Fig. 2. It is clear that, only one peak indicating the direction of uncorrelated interference appears in the spectrum while the directions of the desired signal and the correlated one do not appear. On the other hand, using the modified MUSIC, the spatial spectrum is shown in Fig. 3. It is observed that, three peaks indicating the directions of the three sources are appearing. The estimated DOAs using the modified MUSIC algorithm are applied to the CTMV beam former in order to remove the interference.

The results presented in Fig. 4 show two nulls in the directions of the coherent interference and the uncorrelated interference. The same scenario is repeated in the presence of two uncorrelated interferences at the same aforementioned directions and under the same conditions as the previous case. The results are depicted in Fig.5, which shows two nulls in the directions of the two interferences. Thus, the CTMV is capable of removing either coherent or uncorrelated interferences.

The effect of the interference power on the performance of the proposed system is evaluated. The desired signal is assumed to be either $-5 \mathrm{~dB}$ or $20 \mathrm{~dB}$ above the background noise while the interference power is varying such that the input signal to interference ratio, $\mathrm{SIR}_{\mathbf{i}}$, changes from $-25 \mathrm{~dB}$ to $15 \mathrm{~dB}$. From the combined received signal, 512 snapshots are taken to evaluate the covariance matrix. The modified MUSIC is applied to estimate the DOA of the impinging signals. It is found that the DOA estimation error was typically zero. The output SINR of the CTMV beam former is computed as function of the input SIR. The results are depicted in Fig. 6.

For low power desired signal $\left(\mathrm{SNR}_{\mathrm{i}}=-5 \mathrm{~dB}\right)$, decreasing the interference power improves the output $\mathrm{SINR}_{0}$. This is due to the fact that decreasing the interference power down the signal power level, the noise will be the dominant and the array output will enhance the desired signal. On the other hand for strong desired signal $\left(\mathrm{SNR}_{\mathrm{i}}=20 \mathrm{~dB}\right)$, decreasing the interference power will results in decreasing the output SINR. This is because of the reduction in the DOA estimation accuracy as a result of decreasing the interference power. Consequently the performance of the CTMV is decreased (steered nulls are not in the exact directions of the interference).

\section{4- Conclusions}

In this paper, an adaptive system for nulling coherent and uncorrelated interferences is proposed. The proposed system comprises two units. The first unit combines the preprocessing (spatial smoothing) of impinging signals and the DOA estimation of incident signals. These two operations are implemented by utilizing the modified MUSIC algorithm rather than the traditional MUSIC. The modified MUSIC is used to avoid the failure of the DOA estimation in presence of coherent interference. The second is used for generating nulls in the directions of undesired interferences based on the CTMV method. The objective of the CTMV beam former is to find an adaptive transformation for the covariance matrix of the received signal to another matrix, which does not contain the steering vector of the desired signal. This matrix is used for nulling the spatial spectrum in the directions of undesired interferences while maintain the desired one. The CTMV beam former shows the ability to reduce both coherent and uncorrelated interferences. 
Furthermore, it is found that the performance of CTMV improves as the interference power increases. Now the work is under going to implement a nulling system for wideband interferences.

\section{References}

[1] Don H. Johnson, " Array Signal Processing: Concepts and Techniques", Prentice Hall, PP. 2, 1993.

[2] Joseph C. Liberti, "Smart Antennas for Wireless Communications ", Prentice Hall, PP. 255-270, 1999.

[3] S. U. Pillai, "Performance Analysis of MUSIC Type High Resolution Estimators for Direction Finding in Correlated and Coherent Scenes ", IEEE trans. Signal Processing, Vol. 37. PP. 1176-1189, 1989.

4] Ta-Sung Lee, "Coherent Interference Suppression With complementaryally Transformed Adaptive Beam former ", IEEE trans. Antenna and Propagation, Vol. 46, NO.5, PP. 609617, May 1998.

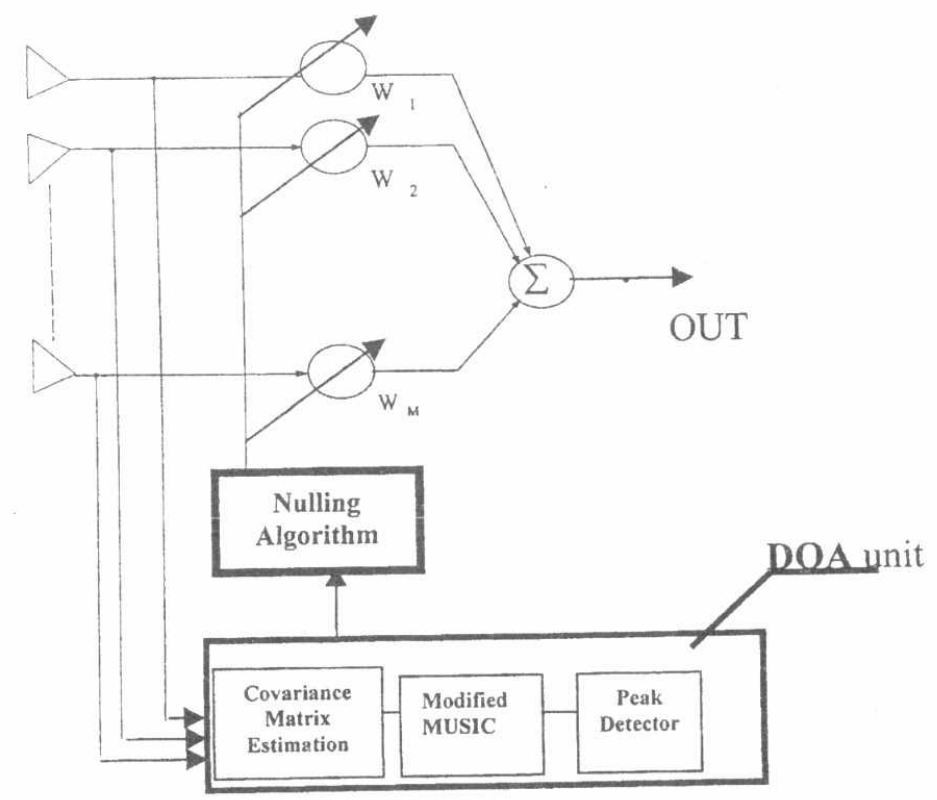

Fig. 1. Structure of the proposed system 


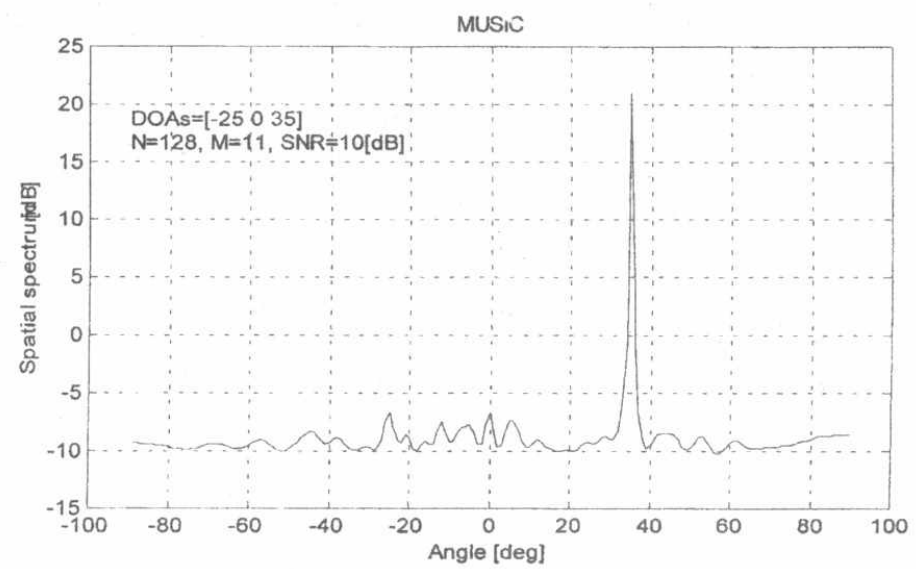

Fig. 2. The spatial spectrum of the MUSIC estimator

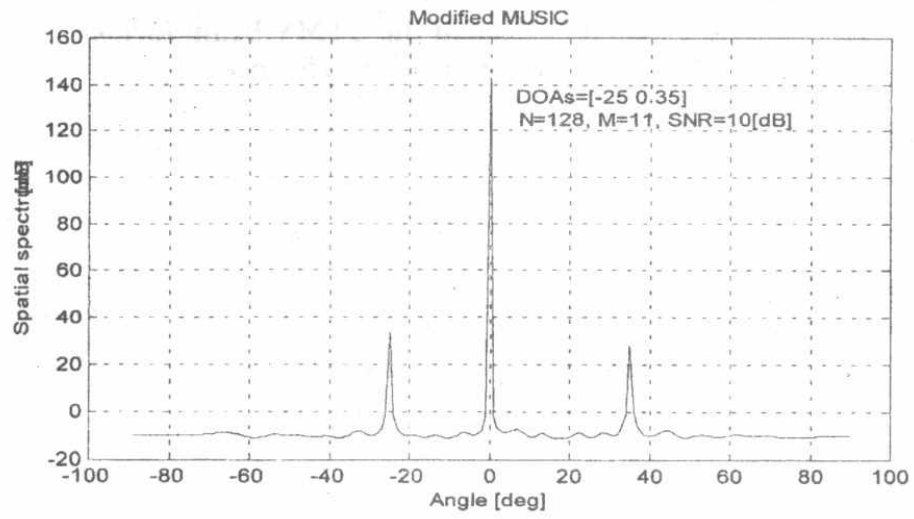

Fig. 3. The spatial spectrum of the Modified MUSIC estimator

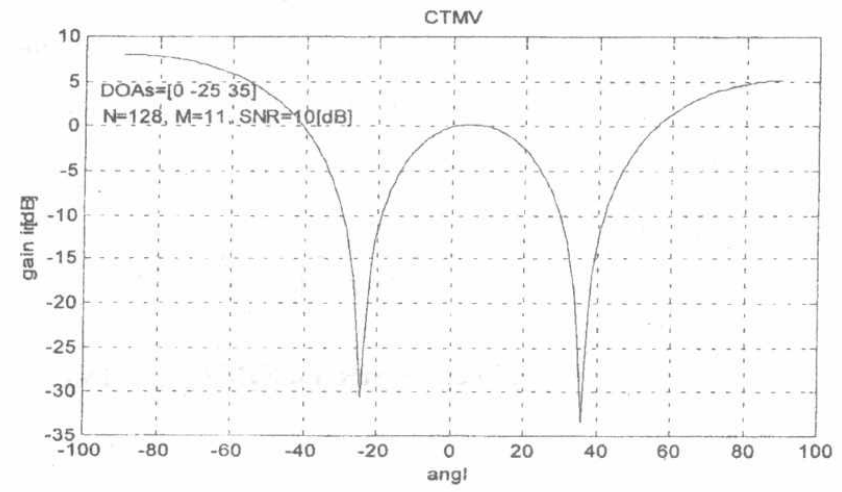

Fig.4. Gain pattern of CTMV beam former (one coherent, one uncorrelated interference) 


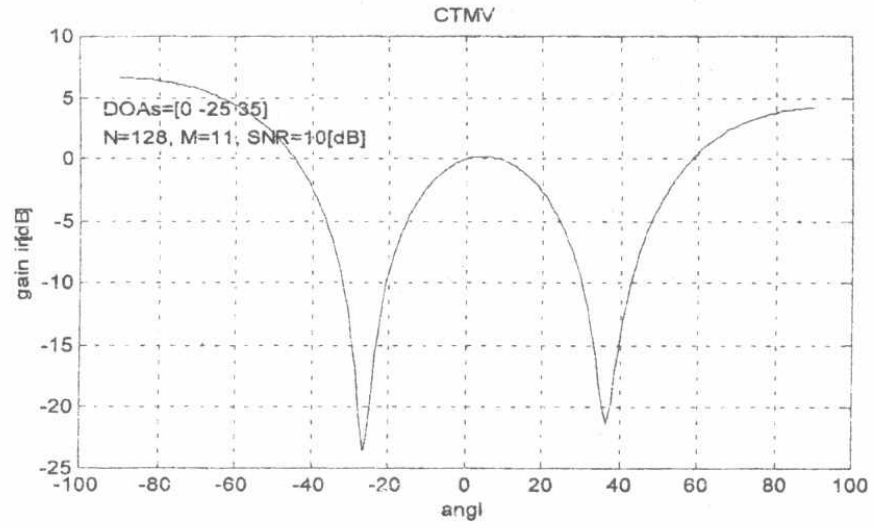

Fig. 5. Gain pattern of the CTMV beam former (two uncorrelated interference)

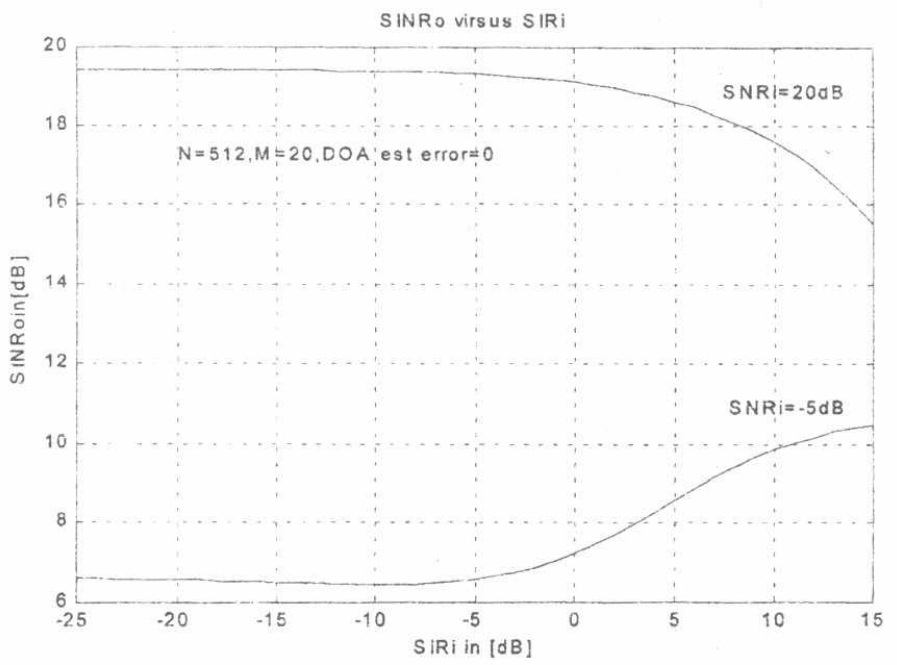

Fig.6 The effect of $\operatorname{SIR}_{i}$ on $\operatorname{SINR}_{0}(C T M V)$ 